\title{
Allergens as Immuno-Modulatory Proteins: the cat dander protein Fel d 1 enhances Toll-like receptor activation by lipid ligands
}

\author{
Jurgen Herre ${ }^{1,2}$, Hans Grönlund ${ }^{3}$, Heather Brooks ${ }^{2}$, Lee Hopkins ${ }^{2}$, Lisa Waggoner ${ }^{4}$, Ben \\ Murton $^{5}$, Monique Gangloff ${ }^{5}$, Olaniyi Opaleye ${ }^{5}$, Edwin R. Chilvers ${ }^{1}$, Kate Fitzgerald ${ }^{4}$, Nick \\ Gay $^{5}$, Tom Monie ${ }^{5, *}$, and Clare Bryant ${ }^{*}$ \\ ${ }_{1}^{1}$ Department of Medicine, The University of Cambridge School of Medicine, Hills Road, \\ Cambridge \\ 2 Department of Veterinary Medicine, University of Cambridge, Madingley Road, Cambridge \\ ${ }^{3}$ Department of Clinical Neuroscience, Karolinska Institute, SE-171 76, Stockholm, Sweden \\ ${ }^{4}$ Department of Medicine, University of Massachusetts Medical School, Worcester MA 01605 \\ ${ }^{5}$ Department of Biochemistry, University of Cambridge, Tennis Court Road, Cambridge
}

\begin{abstract}
Allergic responses can be triggered by structurally diverse allergens. Most allergens are proteins yet extensive research has not revealed how they initiate the allergic response and why the myriad of other inhaled proteins do not. Amongst these allergens, the cat secretoglobulin protein Fel d 1, is the major allergen and responsible for severe allergic responses. In this study we show that like the mite dust allergen Der p 2, Fel d 1 substantially enhances signalling through the innate receptors TLR 4 and TLR2. In contrast to Der $\mathrm{p} 2$ however, Fel $\mathrm{d} 1$ does not act by mimicking the TLR4 co-receptor MD2 and is not able to bind stably to the TLR4/MD2 complex in vitro. Fel d 1 does however, bind to the TLR4 agonist lipopolysaccharide, suggesting that a lipid transfer mechanism may be involved in the Fel d 1 enhancement of TLR signalling. We also show that the dog allergen Can $\mathrm{f} 6$, a member of a distinct class of lipocalin allergens, has very similar properties to Fel d 1 . We propose that Fel $\mathrm{d} 1$ and Can $\mathrm{f} 6$ belong to a group of allergen immunomodulatory proteins (IMPs) that enhance innate immune signalling and promote airway hypersensitivity reactions in diseases such as asthma.
\end{abstract}

\section{Introduction}

The innate immune system is intrinsically linked with allergy. Pattern recognition receptors (PRRs) are involved in allergen sampling, non-specific allergen elimination, and the maintenance of immune tolerance and homeostasis in response to allergens (1). An allergic response can be triggered by many different stimuli, for example: grass pollen, animal dander, foods, insect venoms, pharmaceutical products, chemicals, latex and metals (2). The exact mechanisms by which major allergens are recognized by the host are largely unknown, but recent work suggests that Toll-like receptors (TLRs) play a crucial role in the response to two common allergens, house dust mite protein Der p 2 (3-5) and the metal nickel (6).

"authors for correspondence. Address correspondence and reprint requests to Dr. Tom Monie, Department of Biochemistry, University of Cambridge, 80 Tennis Court Road, Cambridge, CB2 1QW, United Kingdom, and Prof. Clare Bryant, Department of Veterinary Medicine, University of Cambridge, 80 Madingley Road, Cambridge, CB3 OES, United Kingdom. tpm22@cam.ac.uk (T.M.) and ceb27@cam.ac.uk (C.B.). 
Der $\mathrm{p} 2$ is a lipid binding protein that sensitizes ligand-induced signalling through TLR4 and TLR2 $(3,4,7)$. TLR4, in combination with MD2 and CD14, recognizes bacterial lipopolysaccharides (LPS); and TLR2, in a heterodimer with either TLR1 or TLR6, recognizes di- and tri- acylated lipoproteins (8) and lipoteichoic acid (LTA). TLR5 recognises the bacterial protein flagellin $(9,10)$. Ligand recognition by TLRs then activates innate immune signalling pathways (11). Both MD2 and Der $\mathrm{p} 2$ belong to a small family of lipid binding proteins that have a $\beta$-sandwich or cup type fold (12). These proteins recognize lipid by intercalating their acyl chains into the hydrophobic core of the $\beta$-sandwich. Thus, one potential mechanism by which Der $\mathrm{p} 2$ enhances TLR 4 signalling is to mimic MD2 by binding to TLR4. The Der $\mathrm{p}$ 2/TLR4 protein complex may then signal like MD2/TLR4 to activate innate immune signalling (4). In mouse models of allergic asthma the effects of Der p 2 are markedly reduced in TLR4 knockout mice and can be prevented in wild type mice by administration of a TLR4 antagonist (7). House dust mite extracts carrying flagellin can induce TLR5-dependent allergic responses in mice, although the molecular mechanism by which this occurs is unclear (5). Nickel sensitization in humans results from direct, lipid independent activation of TLR4 by $\mathrm{Ni}^{2+}$. Receptor activation is dependent on the presence of two histidine residues, $\mathrm{H} 456$ and $\mathrm{H} 458$, which co-ordinate the $\mathrm{Ni}^{2+}$ atom (or other metal ions such as $\mathrm{Co}^{2+}$ ), promoting TLR4 dimerisation and subsequent receptor activation. Murine TLR4 lacks these histidines and consequently is not activated by nickel $(6,13)$.

Another clinically important allergen is the cat dander protein Fel d 1, which is the commonest cause of severe allergic responses to cats in man (14). In contrast to Der $\mathrm{p} 2$ this allergen has an entirely alpha-helical structure (15) and is thus unlikely to act as a mimetic of MD2. Fel d 1 can bind to the mannose receptor, but immune signalling is not initiated following engagement of this receptor (16). Thus the mechanism by which this protein initiates an allergic response remains unclear.

In this paper we propose a mechanism by which Fel $\mathrm{d} 1$ is recognized by the host to activate immune signalling. Fel d 1 enhances LPS and LTA, but not flagellin-induced TLR signalling. Unlike Der p 2, the mechanism for Fel d 1 enhancement of LPS-induced TLR4/ MD2 activation does not involve the protein binding to the TLRs, but does require the presence of CD14. The dog dander protein Can $\mathrm{f} 6$ (17), a structurally distinct allergen from Fel $\mathrm{d} 1$ and a member of the lipocalin family of allergens, also enhances LPS-induced activation of TLR4 signalling although, unlike Fel d 1, this protein has some MD2 independent effects. We propose, therefore, that animal allergen proteins form a novel class of immune modulator proteins (IMPs) that enhance TLR signalling and hence play a critical role in initiating allergic responses. The mechanism for TLR enhancement of signalling involves formation of a complex of bacterial lipids, such as LPS, with allergen and suggests that inhibitors of TLRs 2 and 4 may represent a new class of therapeutic compounds for the treatment of common allergic diseases.

\section{Materials and Methods}

\section{Protein production}

Fel d 1 was expressed and purified in E. coli. Histidine-tagged and washed inclusion body Fel $\mathrm{d} 1$ was solubilized in guanidine and loaded on a $\mathrm{Ni}^{2+}$ chelate affinity column. The isolated $\mathrm{rFel} \mathrm{d} 1$ preparation was further purified by size exclusion chromatography and equilibrated in PBS. rFel d 1 was subsequently purified from endotoxins on a Detoxi-gelTM (Pierce, Rockford, IL, USA) according to the manufacturer's instructions and stored at $-80^{\circ}$ until required (15).

For expression in baculovirus Gateway Multi-site cloning (Invitrogen) was used to produce an N-terminal RAGE secretion signal followed by Fel d 1 (chains 2+1) and C-terminal V5 
epitope and 6-His tags in a pDEST48 vector (Invitrogen). Bacmids were generated in DH10Bac cells (Invitrogen) following the manufacturer's protocol, purified, and transfected into $s f \rho$ cells to produce a P1 virus stock, which was subsequently amplified and the titre determined to give bFel d 1 virus.

$s f 9$ cells at a density of 1 million / $\mathrm{ml}$ were infected with bFel d 1 virus $(\mathrm{MOI}=1)$ for three days. Clarified supernatants were filtered following supplementation with ammonium sulphate to a final concentration of $300 \mathrm{mM}$. bFel d 1 was recovered using a Butyl-FF column (GE Healthcare) equilibrated in $300 \mathrm{mM}$ ammonium sulphate, $25 \mathrm{mM}$ Tris- $\mathrm{HCl} \mathrm{pH}$ 8. Protein was eluted in $25 \mathrm{mM}$ Tris- $\mathrm{HCl} \mathrm{pH} \mathrm{8.} \mathrm{Fractions} \mathrm{containing} \mathrm{bFel} \mathrm{d} 1$ were pooled and further purified using Ni-NTA resin, before being eluted in $150 \mathrm{mM} \mathrm{NaCl}, 25 \mathrm{mM}$ Tris$\mathrm{HCl} \mathrm{pH} \mathrm{8,} 300 \mathrm{mM}$ imidazole. Eluted fractions were concentrated, and further purified, on an $\mathrm{S} 75$ column that had been washed in $1 \mathrm{M} \mathrm{NaOH}$ and equilibrated in tissue culture grade PBS to minimise LPS contamination. Recombinant Fel d 1 was tested for endotoxin contamination using the Endosafe-PTS assay (Charles-River, UK). This assay system is based upon the Limulus Amebocyte Lysate assay utilizing FDA-licensed disposable cartridges with detection limits from 0.01-10 EU/ml.

Can $\mathrm{f} 6$ was produced as previously described (17). Picia-derived Fel d 1 and Der p 2, as well as natural cat allergen preparations, were from Indoor Biotechnologies, Charlottesville, VA.

\section{Biotinylated LPS pull-down}

Biotinyled Ultrapure E. Coli 011:B4 LPS $1 \mathrm{mg} / \mathrm{ml}$ (InvivoGen) was immobilised on $20 \mu 1$ Strep-Tactin Sepharose bead slurry (IBA). Additional proteins were added to the beads in 10 $\mu \mathrm{l}$ aliquots at $1 \mathrm{mg} / \mathrm{ml}$ concentration and incubated at room temperature with agitation for 20 minutes. Beads were recovered by centrifugation and washed three times in PBS plus $0.05 \%$ Tween20. Beads were boiled in SDS-PAGE sample loading buffer with $5 \mathrm{mM}$ DTT to release bound proteins and the samples analysed by SDS-PAGE.

\section{TLR4/MD2 expression and purification}

Human TLR4 ectodomain (E27-K631) and human MD2 (Q19-N160) fused to a thrombin cleavable Protein A tag were co-expressed in Trichoplusia ni cell culture. The complex was purified via IgG Sepharose 6 (Pharmacia Biotech) affinity purification, followed by on-bead thrombin cleavage, cation exchange and size exclusion through Sepharose 200. The protein was concentrated to $2 \mathrm{mg} / \mathrm{ml}$.

\section{Native PAGE gel}

Purified samples of TLR4/MD2, Fel d 1, CD14, ovalbumin and LPS in PBS were used were used at a concentration of $1 \mathrm{mg} / \mathrm{ml}$. A mixture of $1 \mu \mathrm{l}$ of each component was made and incubated for 30 minutes at room temperature. $1 \mu \mathrm{l}$ of native loading buffer was added to the mixture and $2 \mu \mathrm{l}$ of the final mixture was loaded on to $6 \%$ native-PAGE gel, run and silver stained.

\section{Transient transfection analysis}

HEK293 cells were maintained in Dulbecco's modified Eagle's medium (DMEM) supplemented with $10 \%$ fetal calf serum, $2 \mathrm{mM} \mathrm{L-glutamine,} 100 \mathrm{U} / \mathrm{ml}$ penicillin and $100 \mu \mathrm{g} / \mathrm{ml}$ streptomycin. HEK293 cells were transfected as previously described (18). Briefly cells were seeded at $3 \times 10^{4} /$ well in a 96 well plate and transiently transfected 2 days later. TLR2, TLR4, TLR5 and CD14 were cloned into pcDNA3 and MD2 was sub-cloned into pEFIRES. Expression vectors containing cDNA encoding TLR4, MD2 and CD14 (1 ng/ well of each), a NF-KB transcription reporter vector encoding firefly luciferase (5 ng/well 
pNF-kB-luc, Clontech), and a constitutively active reporter vector encoding Renilla luciferase ( $5 \mathrm{ng} /$ well phRG-TK, Promega), together with empty vector to ensure an optimal amount of DNA were mixed with JetPEI (Polyplus transfection) according to the manufacturer's instructions. TLR2 was co-transfected with CD14 and reporter plasmids. TLR5 was cotransfected with reporter plasmids. After 48 hours cells were stimulated with KDO2-lipidA (a gift from Professor C. Raetz, Duke University, USA) diluted in DMEM supplemented with $0.1 \%$ fetal calf serum in the presence, or absence, of Fel d 1 protein. TNFa stimulation $(1 \mathrm{ng} / \mathrm{ml})$ was used as a positive control. The cells were washed with PBS, lysed, and luciferase activity quantified using the Dual Luciferase kit (Promega) according to the manufacturer's instructions.

\section{Bone Marrow Derived Macrophage stimulation}

Mice were bred under specific pathogen-free conditions at Harlan, UK or the Department of Veterinary Medicine, University of Cambridge, UK. Mice were housed in isolators or in filter-top cages and provided with sterile water and food ad libitum. TLR4-/- mice on a C57BL/6 background were described previously (19). C57BL/6 mice were purchased from Harlan, UK.

BMDMs were isolated from femurs and tibiae of mice killed by cervical dislocation, then cultured in BMDM medium (RPMI1640 medium supplemented with $10 \%$ (v/v) foetal calf serum, $2 \mathrm{mM}$ glutamine, $5 \%$ (v/v) horse serum, $1 \mathrm{mM}$ sodium pyruvate and $10 \mu \mathrm{g} / \mathrm{ml}$ gentamicin), in Petri dishes. For maintenance of BMDMs in culture this medium was further supplemented with $20 \%$ (v/v) of supernatant taken from L929 cells (a murine M-CSFproducing cell line) $(20,21)$. For experiments, cells were plated onto $96-$ well plates at a plating density of $2 \times 10^{5}$ cells per well. Cells were stimulated with ligand in the presence, or absence, of Fel d 1. The small-molecule TLR4 inhibitor CRX-526 (22) was provided by GlaxoSmithKline Vaccines (Hamilton, Montana, USA) as a lyophilized powder. It was resuspended at a concentration of $1 \mathrm{mg} / \mathrm{ml}$ in a diluent of endotoxin-free sterile water containing $2 \%$ glycerol and $0.2 \%$ triethanolamine, at a $\mathrm{pH}$ of $7-7.4$, using a Covaris sonicator and repeated cycles of heating and vortexing. Resuspension was performed at GlaxoSmithKline (Stevenage, UK). The final solution was stored at $4{ }^{\circ} \mathrm{C}$.

\section{Generation of PBMCs}

Human peripheral blood granulocytes were obtained from healthy normal human donors according to the method of Haslett (23) under the study protocol (UK06/Q0108/281) entitled "the inflammatory response of human leucocytes". Briefly, plasma was separated by centrifugation at room temperature and the erythrocyte/leukocyte layer sedimented by the addition of $6 \%$ dextran and diluted with warmed PBS. The suspension was allowed to sediment before the upper leukocyte rich layer was removed and pelleted by centrifugation. The pellet was resuspended in platelet poor plasma and underlayered with freshly prepared Percoll gradient. Following centrifugation the monocyte layer was harvested and further purified with CD14 MACS beads (Miltenyi) as per the manufacturer's protocol. Resultant cells were plated in 96-well plates in RMPI enriched with L-glutamine, Pen-strep, 10 \% FBS and GM-CSF (R\&D; $100 \mathrm{ng} / \mathrm{ml}$ ) and cultured for 7 days before use.

\section{Measurement of cytokine production}

To determine cumulative TNFa production, supernatants were taken at $24 \mathrm{~h}$ post-treatment and stored at $-80^{\circ}$ until analysed with the Duoset ${ }^{\circ}$ ELISA development system (R\&D systems, Abingdon, Oxfordshire, UK). 


\section{Statistics}

Data for HEK transfected cells are presented as representative experiments from an average of at least 3 repeats $(18,24)$. BMDM data is presented as mean data from at least 3 separate biological repeat experiments (25) Graphs were generated using GraphPad Prism and the data analysed using one-way ANOVA and Tukey multiple comparison test for significant differences. Results are expressed as the mean \pm SEM of (n) separate experiments. A P value of $<0.05$ was regarded as significant.

\section{Results}

\section{Fel d 1 enhances lipid induced signalling through both TLR2 and 4}

To determine whether Fel d 1 (like Der p 2) is able to modulate innate immune signalling we expressed and purified recombinant Fel d 1. This protein was made in E. coli and possessed less than $0.5 \mathrm{ng}$ of LPS per mg protein (data not shown). The effect of recombinant Fel d 1 on TLR4/MD2 signalling was tested in a reconstituted HEK293 cell-culture assay. LPS, as expected, induced a concentration dependent increase in relative luciferase activity, but in the presence of Fel d $1(10 \mathrm{ng} / \mathrm{ml})$ the response to LPS was increased by approximately $15-$ fold (Figure 1A). Next we tested whether Fel d 1 also enhanced signalling through TLR2 in response to the ligand LTA. We found that LTA-induced TLR2 signalling was also enhanced in the presence of Fel d 1 (Figure 1B). To rule out the possibility that Fel d 1 enhanced signalling from cell surface receptors in a non-specific manner we carried out equivalent assays with both transiently transfected and endogenous TLR5. Fel d 1 did not modify signalling induced by the TLR5 protein ligand flagellin in either instance (Figure 1C, Supplementary data S1). This suggests that the activity of Fel d 1 to enhance TLR signalling is restricted to those receptors that recognize lipids.

Together these results suggest that animal dander proteins employ a shared mechanism for enhancement of TLR signalling (Figure 6)

\section{Fel d 1 potentiates the production of pro-inflammatory cytokines in primary immune cells}

The recombinant Fel $\mathrm{d} 1$ used in this study causes airway hyper-responsiveness in mice and children by unknown mechanisms (26, 27). To determine whether Fel d 1 enhances innate responses in cells other than transfected HEK293 cells, pro-inflammatory cytokine (TNFa) production was measured from murine bone marrow derived macrophages (BMDM) stimulated with LPS, LTA or the di- and tri-acylated lipopeptides Pam2CSK4 and Pam3CSK4. We required higher concentrations of Fel d 1 to stimulate the murine macrophages compared to the concentration required for activation of the HEK293 cells transfected with TLR4/MD2/CD14. These data are very similar to those from Trompette and colleagues (4), where higher concentrations of Der $\mathrm{p} 2$ were required to activate mouse macrophages than for HEK cells transfected with TLR4/MD2/CD14. Fel d 1 enhanced TNFa production in response to all four bacterial lipid ligands (Figures $2 \mathrm{~A}, \mathrm{~B}$ and $\mathrm{C}$ ). Fel d 1 enhancement of LPS-induced TNFa production was inhibited by the TLR4 antagonist CRX-526, confirming that Fel d 1 sensitises TLR4 signalling in monocyte/macrophage-like cells (Figure 2D). In primary human peripheral blood mononuclear cells (PBMCs) Fel d 1 also enhanced LPS-induced TNFa production in 6 separate donors (Figure 2E). Human cells, as expected, required 5- to 10-fold lower concentrations of LPS for TNFa stimulation in comparison to mouse BMDMs.

In contrast to our E. coli produced recombinant Fel d 1 protein used in these experiments, natural Fel d 1 is glycosylated. A recent study showed that sulphated galactose residues present in these glycans bind to mannose receptors and cause Fel d 1 to be internalized (16). To determine whether the glycosylation status of Fel d 1 influences the sensitization of TLR 
signalling, we compared the properties of a partially glycosylated Fel d 1 produced in the yeast Pichia; glycosylated natural Fel d 1 depleted of LPS; as well as our own Baculovirus produced Fel d 1, in terms of their respective sensitizing effects on TLR4 signalling in BMDMs. These protein preparations all enhanced TLR4 signalling in BMDMs in a similar fashion to the E. coli-derived Fel d 1, showing that the TLR-sensitizing effects of this protein are independent of glycosylation (Figure $2 \mathrm{~F}$ ) and thus mannose receptor activity. Figures 2A, D and F include TLR4 deficient cells as controls. In each case the signal enhancement seen in the presence of Fel d 1 was abolished in TLR4-/- cells, demonstrating that the observed response depends entirely on this receptor.

\section{The enhancement of TLR4 signalling mediated by Fel $d 1$ is dependent on both CD14 and MD2}

We next determined whether, like Der p 2, Fel d 1 could sensitize TLR signalling in the absence of MD2 or CD14. Using HEK293 cells transfected with TLR4 and CD14 in the absence of MD2, we observed that Fel d 1 induced only a small increase in signalling (1.9fold) even at the highest concentration tested (100 ng/ml), compared to a 16-fold increase when MD2 was present (Figure 3A). A similar result was seen when CD14, an extrinsic membrane protein required to deliver LPS to TLR4/MD2, was absent (Figure 3B). These results show that the bioactivity of Fel $\mathrm{d} 1$ in upregulating LPS signalling is dependent on the presence of both MD2 and CD14. These data also show that Fel d 1, unlike Der p2, cannot substitute for MD2 (4) or for CD14.

\section{Fel d 1 does not form a stable complex with TLR4/MD2 but does bind to LPS}

Our data suggest that, unlike Der p 2, Fel d 1 does not mimic MD2 and act as a co-receptor for TLR4, but rather enhances signalling by a different mechanism. One possibility is that this allergen facilitates transfer of LPS to CD14 and MD2. To test this hypothesis we asked first whether either recombinant or natural Fel $\mathrm{d} 1$ is able to form a complex in vitro with TLR4/MD2 or TLR4 alone. To do this we used native polyacrylamide gel electrophoresis and visualized the proteins by silver staining. Fel d 1 preparations were highly pure and showed no contaminating bands (Figure 4A, lane 1; Figure 4B lane 2). Addition of LPS alone to TLR4/MD2 (Figure 4A), or to TLR4 alone (Figure 4B) induced receptor dimerization and oligomerization as shown by changes in the migration of the TLR4 containing species. However, we were unable to observe formation of a complex between recombinant Fel d 1 and TLR4/MD2 (Figure 4A), or natural Fel d 1 and TLR4 (Figure 4B) in either the presence or absence of LPS. Fel d 1 can, however, interact directly with LPS, as streptavadin-coated beads were able to precipitate significant amounts of Fel d 1, but not the control GST, when co-incubated with biotinylated LPS (Figure 4C). Fel d 1 showed no nonspecific binding to the streptavidin-coated beads.

\section{Lipid presentation may be a common mechanism for the action of animal allergens}

Given that both Der p 2 and Fel d 1 enhance TLR signalling, we wondered whether lipid presentation by different allergen proteins could provide a more generic mechanism for animal allergen recognition in the host. To test this hypothesis we generated a structurally unrelated recombinant dog dander allergen, Can $\mathrm{f} 6$ (17), to determine whether this protein could also enhance ligand-induced TLR signalling. Can $\mathrm{f} 6$, like Fel d 1, sensitised TLR4/ MD2/CD14 responses and enhanced LPS-induced signalling in BMDMs (Figure 5A). In contrast, the model allergen OVA (that is not a recognized allergen in humans) had no enhancing effect on TLR4 signalling. Der p 2, as expected, enhanced LPS-induced TLR4 responses albeit to a lesser extent than natural Fel d 1 (Figure 5B). 


\section{Discussion}

Despite Fel d 1 being responsible for approximately $80 \%$ of all human allergic responses to cats, little is known about how it is recognized by the host (2). Here we show for the first time that the major cat and dog allergens, Fel d 1 and Can $\mathrm{f} 6$, cause a substantial amplification of LPS/TLR signalling in both a transfected cell model and in primary, macrophage-like, cells. Importantly, the model allergen OVA, which is not a recognized airways allergen in humans, has no effect on TLR signaling. Unlike the house dust mite allergen Der $\mathrm{p} 2$ these molecules do not act by mimicking the TLR4 co-receptor MD2. Instead they appear to bind microbial lipid PAMPs directly and transfer them to the receptors at the cell surface in a mechanism that depends on CD14. Our work and that of others (4) also shows that, at least in part, Der p 2 also enhances LPS-induced TLR4/MD2 signalling. We propose, therefore, that lipid binding and transfer is a common property of allergen 'immunomodulatory proteins' (IMPs).

In the absence of MD2, a high concentration of Fel d 1 induces a very low level of TLR4/ CD14 activation but even this signal is dependent on CD14. This CD14 and MD2 dependence indicates that Fel $\mathrm{d} 1$ does not work mechanistically by substituting for their functions. This, along with the fact that Fel d 1 also enhances LTA-induced activation of TLR2, suggests that the IMPs may be increasing the availability of lipids to CD14 and the TLR signalling complex. Alternatively, Fel d 1 may facilitate the assembly of TLR signalling complexes in membrane microdomains thus lowering the activation threshold (28) (Figure 6).

Although the IMPs appear to have a similar mechanism for enhancing innate immune signalling they all have very diverse 3-dimensional structures. Der $\mathrm{p} 2$ is a member of a small family of lipid binding proteins and has a similar $\beta$-cup structure to MD2. In the Der $p$ 2 crystal structure electron density can be seen that most likely corresponds to at least one fatty acyl chain and by comparison with MD2 it is likely that this molecule can accommodate a hexa-acyl glycolipid like LPS $(29,30)$. Fel d 1 , on the other hand, is a heterodimer of two related chains that forms a structure with 8 a-helices stabilized by intramolecular disulphide bonds. The subunit interface forms a hydrophobic cavity that may represent the binding site for microbial lipid ligands of the TLRs. The third IMP we have studied is the newly described Can $\mathrm{f}$, which causes sensitization in $35 \%$ of patients allergic to dogs. It is a lipocalin allergen, a family that also includes $\operatorname{dog} \operatorname{Can} \mathrm{f} 1$, Can $\mathrm{f} 2$, Can $\mathrm{f} 4$, cat Fel d 4 and Equ c 1 from the horse (17). Lipocalins form an 8-stranded $\beta$-barrel structure with a hydrophobic cavity to which small lipophilic molecules, such as pheromones, can bind (31). It is probable that, like Der $\mathrm{p} 2$ and Fel d 1, these allergens will bind to the lipid ligands of the TLRs.

Previous studies showed that TLR4 in particular is required to develop allergic responses to Der $\mathrm{p} 2$, at least in a mouse model of asthma. These studies also showed that TLR4 function is likely to be required not only in innate immune cells but also in the airway epithelia (7). Sensitization to inhaled allergens is caused by the generation of allergen-specific IgE antibody response and several epitopes have been defined in Fel d 1 and other IMPs (15, 17). To generate an antibody response the allergen must be taken up and presented by dendritic cells in a $\mathrm{T}_{\mathrm{H}} 2$ polarizing cytokine environment. In the case of Fel d 1 uptake by dendritic cells may be mediated by cell surface mannose receptors (16) but this process appears to be independent of TLR2/4 activation (Figure 3). A possible hypothesis for allergen action is that IMPs stimulate TLR signalling in the airway epithelium leading to the production of $\mathrm{T}_{\mathrm{H}} 2$ cytokines, such as IL-4 and IL-13 $(5,32)$. TLR signalling might also undermine the barrier function of the epithelium allowing allergens to access innate cells within the lamina propria $(33,34)$. In this regard it is known that the protein kinase $\mathrm{C} \delta$ 
isoform and myosin light chain kinase are activated by the TLR2 and TLR4 (35). These kinases can promote the disassembly of tight junctions by phosphorylating regulatory molecules. In hypersensitivity responses it is possible that activation of TLRs by allergens also increases the permeability of the respiratory epithelia, allowing access to allergen specific IgE. Aggregates of IgE and IMPs would then ligate Fce.receptors leading to activation of mast cells and rapid release of inflammatory mediators.

In conclusion we have shown that a number of mammalian IMPs enhance TLR signalling in response to lipid ligands. Agonists and antagonists to TLRs, therefore, may provide new therapeutic targets to modulate and treat allergic responses to animal-derived allergens.

\section{Supplementary Material}

Refer to Web version on PubMed Central for supplementary material.

\section{Acknowledgments}

We would like to thank Professor S. Akira for provision of the TLR4 -/- mice.

Funding: This work was supported by a program grant from the Wellcome Trust (081744/z/o6/z wt) and MRC (G1000133) to NJG and CEB. CEB is supported by a BBSRC Research Development Fellowship. TPM is supported by a Wellcome Trust Career Development Fellowship (WT085090MA). JH was supported by an Academy of Medical Sciences Starter Grant for Clinical Lecturers.

\section{References}

1. Minnicozzi M, Sawyer RT, Fenton MJ. Innate immunity in allergic disease. Immunological reviews. 2011; 242:106-127. [PubMed: 21682741]

2. Spitzauer S. Allergy to mammalian proteins: at the borderline between foreign and self? International archives of allergy and immunology. 1999; 120:259-269. [PubMed: 10640909]

3. Chiou Y-L, Lin C-Y. Der p2 activates airway smooth muscle cells in a TLR2/MyD88-dependent manner to induce an inflammatory response. Journal of cellular physiology. 2009; 220:311-318. [PubMed: 19326394]

4. Trompette A, Divanovic S, Visintin A, Blanchard C, Hegde RS, Madan R, Thorne PS, Wills-Karp M, Gioannini TL, Weiss JP, Karp CL. Allergenicity resulting from functional mimicry of a Toll-like receptor complex protein. Nature. 2009; 457:585-588. [PubMed: 19060881]

5. Wilson RH, Maruoka S, Whitehead GS, Foley JF, Flake GP, Sever ML, Zeldin DC, Kraft M, Garantziotis S, Nakano H, Cook DN. The Toll-like receptor 5 ligand flagellin promotes asthma by priming allergic responses to indoor allergens. Nature medicine. 2012; 18:1705-1710.

6. Schmidt M, Raghavan B, Müller V, Vogl T, Fejer G, Tchaptchet S, Keck S, Kalis C, Nielsen PJ, Galanos C, Roth J, Skerra A, Martin SF, Freudenberg M. a, Goebeler M. Crucial role for human Toll-like receptor 4 in the development of contact allergy to nickel. Nature immunology. 2010; 11:814-819. [PubMed: 20711192]

7. Hammad H, Chieppa M, Perros F, Willart MA, Germain RN, Lambrecht BN. House dust mite allergen induces asthma via Toll-like receptor 4 triggering of airway structural cells. Nature medicine. 2009; 15:410-416.

8. Kang JY, Nan X, Jin MS, Youn S-J, Ryu YH, Mah S, Han SH, Lee H, Paik S-G, Lee J-O. Recognition of lipopeptide patterns by Toll-like receptor 2-Toll-like receptor 6 heterodimer. Immunity. 2009; 31:873-884. [PubMed: 19931471]

9. Gewirtz AT, Navas TA, Lyons S, Godowski PJ, Madara JL. Cutting edge: bacterial flagellin activates basolaterally expressed TLR5 to induce epithelial proinflammatory gene expression. Journal of immunology (Baltimore, Md. : 1950). 2001; 167:1882-1885.

10. Hayashi F, Smith KD, Ozinsky A, Hawn TR, Yi EC, Goodlett DR, Eng JK, Akira S, Underhill $\mathrm{DM}$, Aderem a. The innate immune response to bacterial flagellin is mediated by Toll-like receptor 5. Nature. 2001; 410:1099-1103. [PubMed: 11323673] 
11. Bryant C, Fitzgerald K. Molecular mechanisms involved in inflammasome activation. Trends in cell biology. 2009; 19:455-464. [PubMed: 19716304]

12. Inohara N, Nuñez G. ML -- a conserved domain involved in innate immunity and lipid metabolism. Trends in biochemical sciences. 2002; 27:219-221. [PubMed: 12076526]

13. Raghavan B, Martin SF, Esser PR, Goebeler M, Schmidt M. Metal allergens nickel and cobalt facilitate TLR4 homodimerization independently of MD2. EMBO reports. 2012; 13:1109-1115. [PubMed: 23059983]

14. Lowenstein H, Lind P, Weeke B. Identification and clinical significance of allergenic molecules of cat origin. Part of the DAS 76 study. Allergy. 1985; 40:430-431. [PubMed: 4051144]

15. Kaiser L, Grönlund H, Sandalova T, Ljunggren H-G, van Hage-Hamsten M, Achour A, Schneider G. The crystal structure of the major cat allergen Fel d 1, a member of the secretoglobin family. The Journal of biological chemistry. 2003; 278:37730-37735. [PubMed: 12851385]

16. Emara M, Royer P-J, Abbas Z, Sewell HF, Mohamed GG, Singh S, Peel S, Fox J, Shakib F, Martinez-Pomares L, Ghaemmaghami AM. Recognition of the major cat allergen Fel d 1 through the cysteine-rich domain of the mannose receptor determines its allergenicity. The Journal of biological chemistry. 2011; 286:13033-13040. [PubMed: 21335554]

17. Nilsson OB, Binnmyr J, Zoltowska A, Saarne T, van Hage M, Grönlund H. Characterization of the dog lipocalin allergen Can $\mathrm{f} 6$ : the role in cross-reactivity with cat and horse. Allergy. 2012; 67:751-757. [PubMed: 22515174]

18. Walsh C, Gangloff M, Monie T, Smyth T, Wei B, McKinley TJ, Maskell D, Gay N, Bryant C. Elucidation of the MD-2/TLR4 interface required for signaling by lipid IVa. Journal of immunology (Baltimore, Md. : 1950). 2008; 181:1245-1254.

19. Hoshino K, Takeuchi O, Kawai T, Sanjo H, Ogawa T, Takeda Y, Takeda K, Akira S. Cutting edge: Toll-like receptor 4 (TLR4)-deficient mice are hyporesponsive to lipopolysaccharide: evidence for TLR4 as the Lps gene product. Journal of immunology (Baltimore, Md. : 1950). 1999; 162:3749_ 3752.

20. Royle MCJ, Tötemeyer S, Alldridge LC, Maskell DJ, Bryant CE. Stimulation of Toll-like receptor 4 by lipopolysaccharide during cellular invasion by live Salmonella typhimurium is a critical but not exclusive event leading to macrophage responses. Journal of immunology (Baltimore, Md. : 1950). 2003; 170:5445-5454.

21. Yamamoto-Yamaguchi Y, Tomida M, Hozumi M. Effect of mouse interferon on growth and differentiation of mouse bone marrow cells stimulated by two different types of colony-stimulating factor. Blood. 1983; 62:597-601. [PubMed: 6603878]

22. Bazin HG, Murray TJ, Bowen WS, Mozaffarian A, Fling SP, Bess LS, Livesay MT, Arnold JS, Johnson CL, Ryter KT, Cluff CW, Evans JT, Johnson DA. The "Ethereal" nature of TLR4 agonism and antagonism in the AGP class of lipid A mimetics. Bioorganic \& medicinal chemistry letters. 2008; 18:5350-5354. [PubMed: 18835160]

23. Haslett C, Guthrie LA, Kopaniak MM, Johnston RB, Henson PM. Modulation of multiple neutrophil functions by preparative methods or trace concentrations of bacterial lipopolysaccharide. The American journal of pathology. 1985; 119:101-110. [PubMed: 2984939]

24. Rallabhandi P, Awomoyi A, Thomas KE, Phalipon A, Fujimoto Y, Fukase K, Kusumoto S, Qureshi N, Sztein MB, Vogel SN. Differential activation of human TLR4 by Escherichia coli and Shigella flexneri 2a lipopolysaccharide: combined effects of lipid A acylation state and TLR4 polymorphisms on signaling. Journal of immunology (Baltimore, Md. : 1950). 2008; 180:11391147.

25. Talbot S, Tötemeyer S, Yamamoto M, Akira S, Hughes K, Gray D, Barr T, Mastroeni P, Maskell DJ, Bryant CE. Toll-like receptor 4 signalling through MyD88 is essential to control Salmonella enterica serovar typhimurium infection, but not for the initiation of bacterial clearance. Immunology. 2009; 128:472-483. [PubMed: 19930040]

26. Saarne T, Neimert-Andersson T, Grönlund H, Jutel M, Gafvelin G, van Hage M. Treatment with a Fel d 1 hypoallergen reduces allergic responses in a mouse model for cat allergy. Allergy. 2011; 66:255-263. [PubMed: 20804464]

27. Grönlund H, Adédoyin J, Reininger R, Varga E-M, Zach M, Fredriksson M, Kronqvist M, Szepfalusi Z, Spitzauer S, Grönneberg R, Valenta R, Hedlin G, van Hage M. Higher 
immunoglobulin E antibody levels to recombinant Fel d 1 in cat-allergic children with asthma compared with rhinoconjunctivitis. Clinical and experimental allergy : journal of the British Society for Allergy and Clinical Immunology. 2008; 38:1275-1281. [PubMed: 18477016]

28. Triantafilou M, Morath S, Mackie A, Hartung T, Triantafilou K. Lateral diffusion of Toll-like receptors reveals that they are transiently confined within lipid rafts on the plasma membrane. Journal of cell science. 2004; 117:4007-4014. [PubMed: 15286178]

29. Derewenda U, Li J, Derewenda Z, Dauter Z, Mueller GA, Rule GS, Benjamin DC. The crystal structure of a major dust mite allergen Der $\mathrm{p} 2$, and its biological implications. Journal of molecular biology. 2002; 318:189-197. [PubMed: 12054778]

30. Park BS, Song DH, Kim HM, Choi B-S, Lee H, Lee J-O. The structural basis of lipopolysaccharide recognition by the TLR4-MD-2 complex. Nature. 2009; 458:1191-1195. [PubMed: 19252480]

31. Madhurantakam C, Nilsson OB, Uchtenhagen H, Konradsen J, Saarne T, Högbom E, Sandalova T, Grönlund H, Achour A. Crystal structure of the dog lipocalin allergen Can f 2: implications for cross-reactivity to the cat allergen Fel d 4. Journal of molecular biology. 2010; 401:68-83. [PubMed: 20621650]

32. Mukherjee S, Chen L-Y, Papadimos TJ, Huang S, Zuraw BL, Pan ZK. Lipopolysaccharide-driven Th2 cytokine production in macrophages is regulated by both MyD88 and TRAM. The Journal of biological chemistry. 2009; 284:29391-29398. [PubMed: 19638630]

33. Gregory LG, Lloyd CM. Orchestrating house dust mite-associated allergy in the lung. Trends in immunology. 2011; 32:402-411. [PubMed: 21783420]

34. Marchiando AM, Graham WV, Turner JR. Epithelial barriers in homeostasis and disease. Annual review of pathology. 2010; 5:119-144.

35. Kubo-Murai M, Hazeki K, Sukenobu N, Yoshikawa K, Nigorikawa K, Inoue K, Yamamoto T, Matsumoto M, Seya T, Inoue N, Hazeki O. Protein kinase Cdelta binds TIRAP/Mal to participate in TLR signaling. Molecular immunology. 2007; 44:2257-2264. [PubMed: 17161867] 

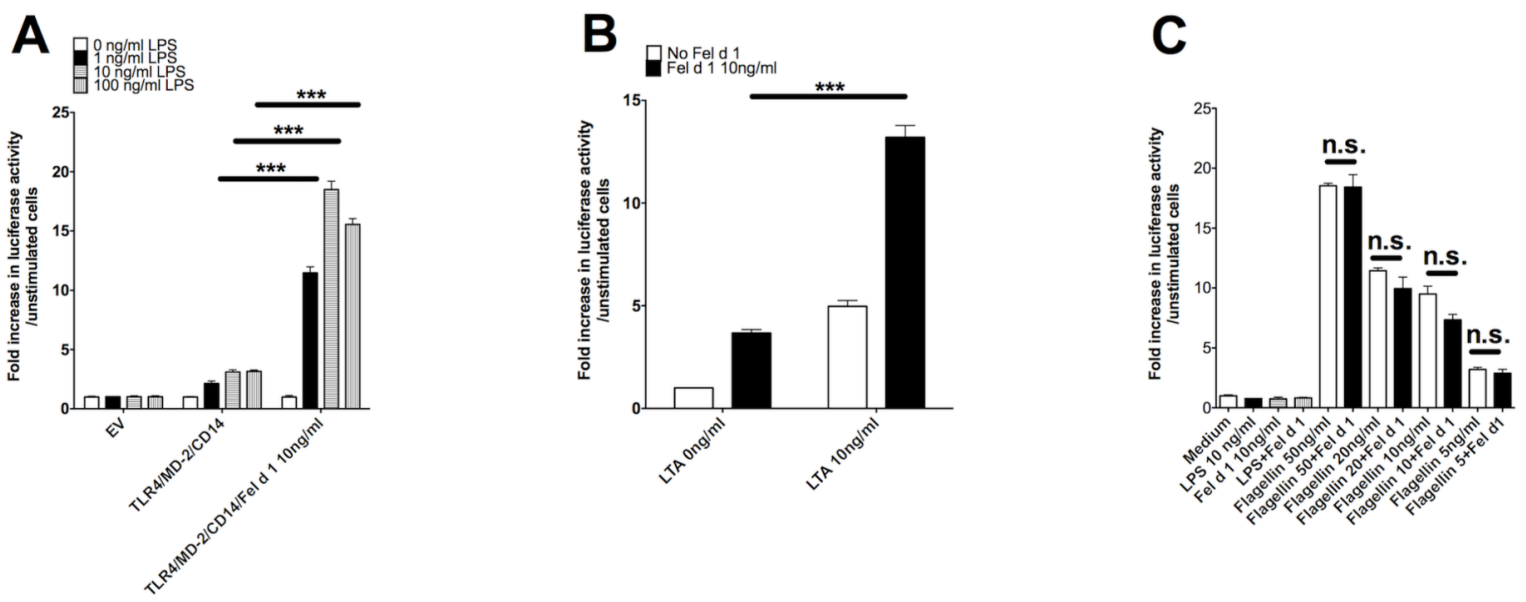

Figure 1. Fel d 1 enhances signalling by ligands of TLR2 and 4 but not TLR5

(A) HEK293 cells transiently transfected to express TLR4, CD14, MD2, an NFkB-luciferase reporter (pNFkB-luc) and a constitutively active luciferase reporter control (phRG-TK) were treated for 6 hours with LPS (1-100 ng/ml) in the presence or absence of Fel d $1(10 \mathrm{ng} / \mathrm{ml})$. Cells were lysed and the NFkB luciferase reporter was read on a FLUOstar Omega (BMG Labtech) luminometer. Data are normalized against the unstimulated control. The graph is one representative experiment from 4 separate experiments. (B) HEK293 cells transiently transfected with TLR2, CD14 pNFkB-luc and phRG-TK were stimulated with lipotechoic acid (LTA) at 10ng/ml in the presence, and absence, of Fel d 1 (10 ng/ml). Data are normalized against the unstimulated control. Data are shown from one representative experiment from 3 separate experiments. (C) HEK293 cells were transiently transfected with TLR5, NFkB-luc and phRG-TK) then stimulated with flagellin $(5-50 \mathrm{ng} / \mathrm{ml})$ in the presence or absence of Fel d $1(10 \mathrm{ng} / \mathrm{ml})$. Data normalized against unstimulated control are shown as one representative experiment from 5 separate experiments. Data represent mean and s.e.m. of a representative experiment from $4-5$ repeat experiments. $* \mathrm{P}<0.05$; $* * \mathrm{P}<0.005 ; * * * \mathrm{P}<0.001 ;$ n.s., not significant. 
A
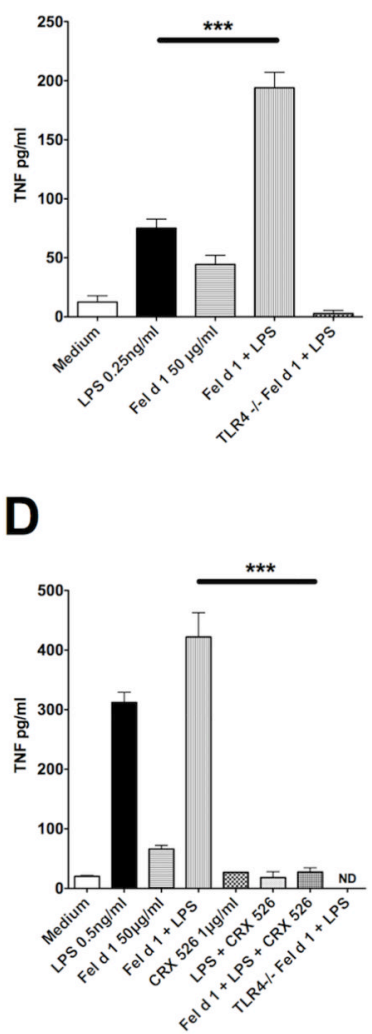

B
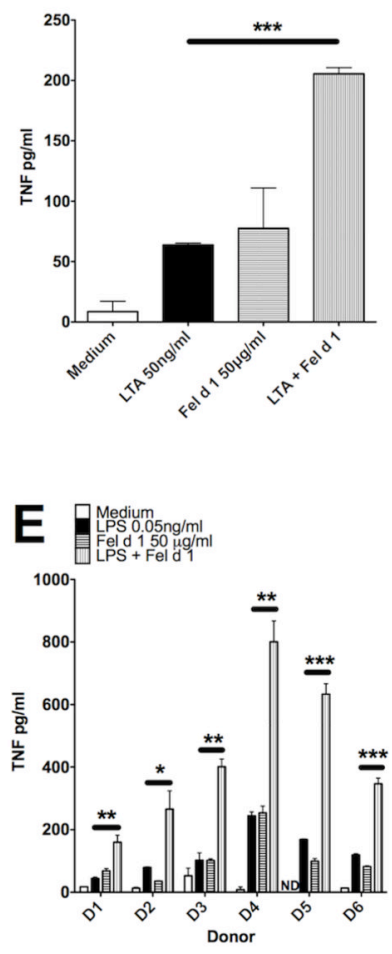

C

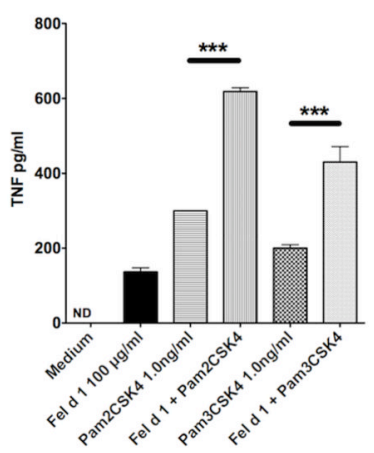

$\mathbf{F}$

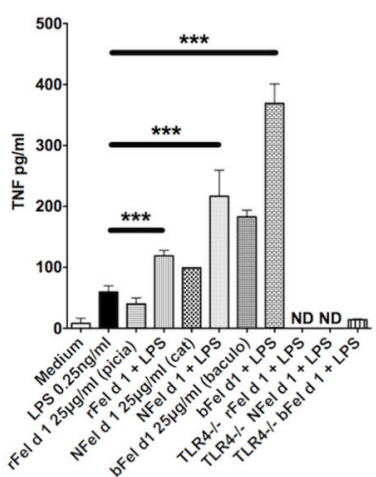

Figure 2. Fel d 1 enhances expression of the pro-inflammatory cytokine TNF-alpha in primary cells of the innate immune system

(A) Bone marrow derived macrophages (BMDM) from wild type and TLR4-/- C57BL6 mice were treated for $24 \mathrm{~h}$ with LPS at $0.5 \mathrm{ng} / \mathrm{ml}$, Fel d 1 at $50 \mu \mathrm{g} / \mathrm{ml}$ or both, as indicated for 24 hours. TNF-alpha levels were measured by an ELISA (R\&D). Data from 5 separate experiments was pooled and expressed as mean \pm sem. (B) Wild type BMDMs were treated with LTA at $50 \mathrm{ng} / \mathrm{ml}$ with or without Fel d 1 at $50 \mu \mathrm{g} / \mathrm{ml}$ for 24 hours. TNF-alpha levels were measured by an ELISA (R\&D). Data from 3 separate experiments were pooled and expressed as mean \pm sem. (C) Wild-type BMDMs treated for $24 \mathrm{~h}$ with di- and tri acylated lipid activators of TLR1/2 and TLR2/6 (Pam3CSK4 1.0ng/ml and Pam2CSK4 1.0ng/ml) with or without Fel d 1 at $100 \mu \mathrm{g} / \mathrm{ml}$. TNF-alpha levels were measured by an ELISA (R\&D). Data from 2 separate experiments were pooled and expressed as mean \pm sem. (D) Wild type and TLR4-/- BMDMs were pre-treated with the TLR4 small molecule inhibitor CRX-526 for 60 minutes and then treated for $24 \mathrm{~h}$ with LPS $0.5 \mathrm{ng} / \mathrm{ml}$ with or without Fel d 1 at $50 \mu \mathrm{g} /$ ml. CRX-526 was maintained throughout the stimulation period. TNF-alpha levels were measured by an ELISA (R\&D). Data from 2 separate experiments were pooled and expressed as mean \pm sem. (E) Peripheral Blood Mononuclear Cells (PBMC) derived from healthy donors as part of an ethically approved research programme were treated for $24 \mathrm{~h}$ with LPS $0.05 \mathrm{ng} / \mathrm{ml}$ with or without Fel d 1 at $50 \mu \mathrm{g} / \mathrm{ml}$. Donors are shown separately to demonstrate inter individual variation. TNF-alpha levels were measured by an ELISA (R\&D). Data are shown from 3 healthy donors. (F) BMDM from wild type and TLR4-/mice treated with LPS $0.25 \mathrm{ng} / \mathrm{ml}$ and six alternative sources of Fel d 1 (recombinant protein 
expressed in the yeast Pichia pastoris ( $\mathrm{rFel} \mathrm{d} \mathrm{1),} \mathrm{natural} \mathrm{protein} \mathrm{from} \mathrm{the} \mathrm{cat} \mathrm{depleted} \mathrm{of} \mathrm{LPS}$

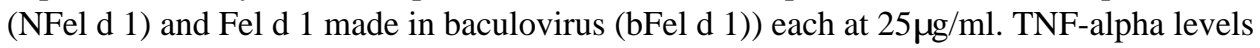
were measured by an ELISA (R\&D). Data from 3 separate experiments were pooled and expressed as mean \pm sem. $* \mathrm{P}<0.05 ; * * \mathrm{P}<0.005 ; * * * \mathrm{P}<0.001 ;$ n.s., not significant 

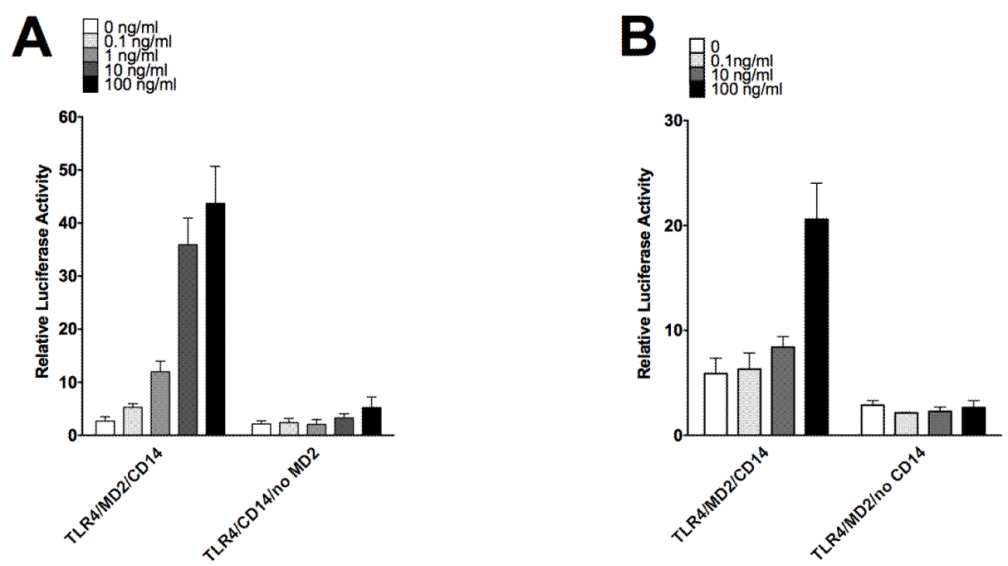

Figure 3. Signal enhancement by Fel d 1 requires the TLR4 co-receptor MD2 and CD14 (A) HEK293 cells were transfected with components of the TLR4 signalling complex: either TLR4, MD2 and CD14; or TLR4 and CD14, an NFkB-luciferase reporter (pNFkB-luc) and a constitutively active luciferase reporter control (phRG-TK) and then stimulated with Fel d $1(0.1-100 \mathrm{ng} / \mathrm{ml})$. The graph is one representative experiment from 3 separate experiments. (B) HEK293 cells were transfected with components of the TLR4 signalling complex: either TLR4, MD2 and CD14; or TLR4 and MD2 an NFkB-luciferase reporter (pNFkB-luc) and a constitutively active luciferase reporter control (phRG-TK) and then stimulated with Fel d 1 $(0.1-100 \mathrm{ng} / \mathrm{ml})$. The graph is one representative experiment from 3 separate experiments. 


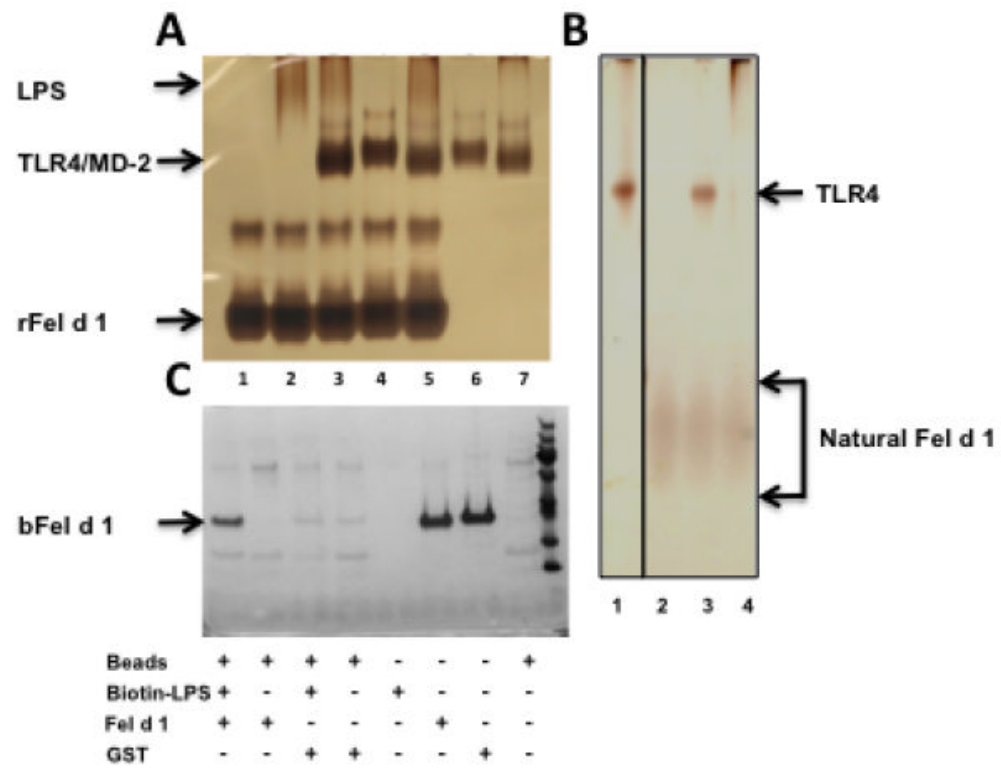

Figure 4. Fel d 1 interacts with LPS, but not with the TLR4/MD2 complex

(A) Recombinant Fel d 1 does not interact with TLR4/MD2. Silver-stained gel of recombinant Fel d 1 (rFel d 1) that has been incubated with LPS and components of a copurified TLR4/MD2 complex. $1 \mu \mathrm{g}$ of each component was added in the order listed and incubated at room temperature for $30 \mathrm{~min}: 1=\mathrm{rFel} \mathrm{d}$ 1alone; $2=\mathrm{rFel} \mathrm{d} 1+\mathrm{LPS} ; 3=\mathrm{rFel} \mathrm{d}$ 1 + LPS + TLR4/MD2; 4 =rFel d 1 + TLR4/MD2; $5=$ rFel d 1 + TLR4/MD2 + LPS; $6=$ TLR4/MD2; 7 = TLR4/MD2 + LPS. The position of migration for each component is shown. rFel d 1 migrates as two bands consistent with the formation of a dimer and tetramer. (B) Natural Fel d 1 does not form a complex with TLR4 either in the presence or absence of LPS. $1 \mu \mathrm{g}$ of each of the listed components were incubated together at room temperature for 30 minutes: 1 = TLR4 alone; 2 = natural Fel d 1 alone; 3 = natural Fel d 1 + TLR4; $4=$ natural Fel d 1 + LPS + TLR4. No shift in the band for natural Fel d 1 was observed. (C) Fel d 1 interacts with LPS. Recombinant Fel d 1 from baculovirus (bFel d 1) was incubated with biotin-LPS and the complex pulled down using streptavidin-conjugated beads (lane 1). The position of bFel d 1 is marked. Lanes 2, 3 and 4 control for non-specific binding of bFel d 1 to the streptavidin-coated beads; non-specific binding of proteins to biotin-LPS; and nonspecific binding of GST to the streptavidin-coated beads. Lanes 5 to 8 indicate assay inputs. 


\section{A}

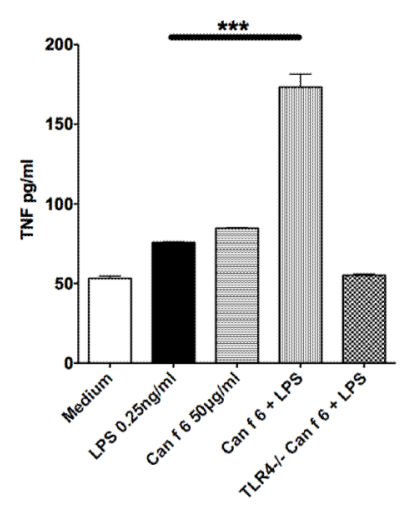

B

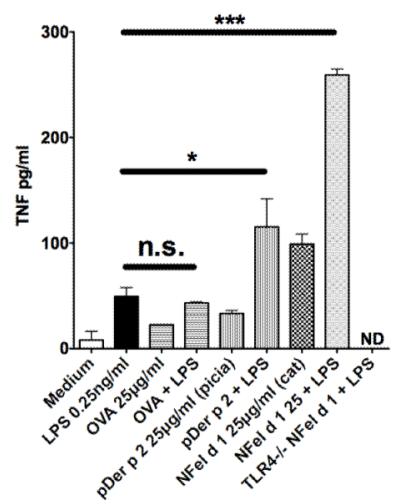

Figure 5. Allergens from three different structural classes sensitize signalling by TLR4 and TLR2

(A) Wild-type and TLR4-/- BMDM treated with LPS at $0.25 \mathrm{ng} / \mathrm{ml}$ in the presence, or absence, of the dog lipocalin allergen Can $\mathrm{f} 6$ at $50 \mu \mathrm{g} / \mathrm{ml}$ for 24 hours. TNF-alpha levels were measured by an ELISA (R\&D). Data from 3 separate experiments were pooled and expressed as mean \pm sem. (B) Wild-type and TLR4-/- BMDMs treated with LPS (0.25ng/ $\mathrm{ml})$, the house dust mite allergen Der p $2(25 \mu \mathrm{g} / \mathrm{ml})$, the model allergen protein ovalbumin $(\mathrm{OVA}, 20 \mu \mathrm{g} / \mathrm{ml})$ or cat derived Fel d 1 (NFel d 1, 25 $\mu \mathrm{g} / \mathrm{ml})$ either singly or in combination for $24 \mathrm{~h}$. TNF-alpha levels were measured by an ELISA (R\&D). Data from 3 separate experiments were pooled and expressed as mean \pm sem. $* \mathrm{P}<0.05 ; * * \mathrm{P}<0.005 ; * * * \mathrm{P}<0.001$; n.s., not significant. 


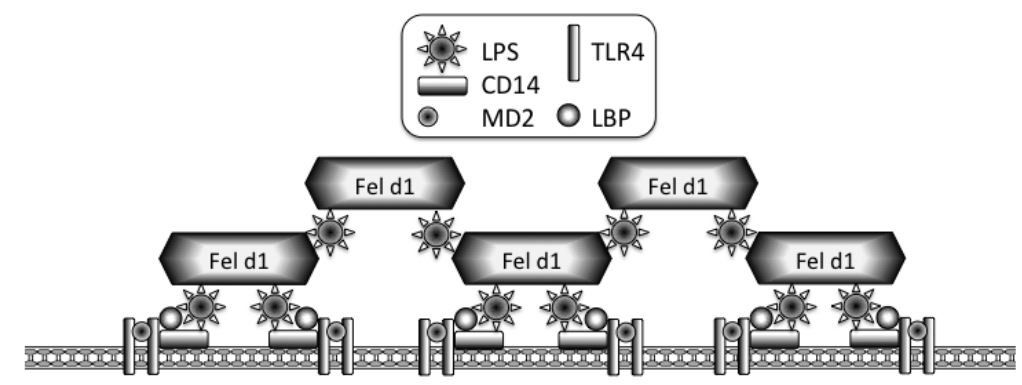

Figure 6. Model of LPS sensitization by dander proteins

Animal dander proteins loaded with environmentally derived lipid pathogen-associated molecular patterns (PAMPs) associate with cell membrane, facilitating lipid presentation or transfer to receptor complexes. Dander proteins, in the presence of low LPS concentrations, could cluster together with LPS to form larger complexes that then promote greater clustering of TLR4 bearing lipid rafts, leading to increased receptor activation. At higher LPS exposure levels (such as would not be seen from environmental sources), this effect is not seen because maximal receptor activation has already been achieved. 